\title{
$X$-INNER AUTOMORPHISMS OF FILTERED ALGEBRAS
}

\author{
SUSAN MONTGOMERY ${ }^{1}$
}

\begin{abstract}
For the enveloping algebra of a finite-dimensional Lie algebra, and for the ring of differential polynomials over a commutative domain, we compute the group of those automorphisms which become inner when extended to the quotient division rings. Both of these results depend on a more general result about the automorphisms of a filtered algebra.
\end{abstract}

Let $A$ be a prime ring. An $X$-inner automorphism $\sigma$ of $A$ is one which becomes inner when extended to the Martindale quotient ring $A_{\mathscr{G}}$ of $A$; when $A$ is a prime Goldie ring, this condition is equivalent to $\sigma$ becoming inner on the classical quotient ring of $A$ [5]. Thus the set of all $X$-inner automorphisms is a normal subgroup of $\operatorname{Aut}(A)$ which contains all the inner automorphisms; this set has proved useful in studying group actions on rings and crossed products. Recently the $X$-inner automorphisms have been computed for certain group rings [7] and for coproducts of domains [4].

In this note we consider a filtered algebra $A$ such that the associated graded ring $\bar{A}$ is a commutative domain. We show that any $X$-inner automorphism of $A$ preserves the filtration of $A$ and induces the trivial automorphism on $\bar{A}$. We then give two applications of this result: we determine the $X$-inner automorphisms of $U(\mathrm{~g})$, the enveloping algebra of a finite-dimensional Lie algebra $\mathrm{g}$, and also of the differential polynomial ring $A=R[x ; d]$, where $R$ is a commutative domain with nontrivial derivation $d$.

We require some definitions. For a prime ring $A$, the Martindale quotient ring of $A$ is the left quotient ring of $A$ with respect to the filter $\mathscr{F}$ of all nonzero two-sided ideals; that is $A_{\mathscr{F}}=\lim _{I \in \mathscr{F}} \operatorname{Hom}_{A}\left({ }_{A} I, A\right) . A_{\mathscr{G}}$ is also a prime ring, containing $A$, and for any $0 \neq x \in A$, there exists $I \in \mathscr{F}$ so that $0 \neq I x \subseteq A$ (for details see [3]). Moreover, for any $\sigma \in \operatorname{Aut}(A), \sigma$ has a unique extension to $A_{\mathscr{F}}$

Now assume that $\sigma$ is $X$-inner. That is, for some $a \in A_{\mathscr{F}}, r^{\sigma}=a^{-1} r a$, all $r \in A$. Let $I \in \mathcal{F}$ be such that $0 \neq I a \subseteq A$, and choose $b, c \in A$ with $0 \neq b a=c$. Then for any $r \in R, c(r b)^{\sigma}=b a(r b)^{\sigma}=b(r b) a=b r c$. That is,

$$
b r c=c r^{\circ} b^{\circ}, \text { all } r \in A .
$$

Conversely, it is not difficult to show that if for some $\sigma \in \operatorname{Aut}(A)$, there exist $0 \neq b, c \in A$ such that $b r c=c r^{\sigma} b^{\sigma}$, all $r \in A$, then $\sigma$ is $X$-inner [6].

Now let $A$ be a filtered ring, say $A=\cup_{n>0} A_{n}$, where $1 \in A_{0}$. For any $a \in A$, let $f(a)$ denote the filtration of $a$; that is, $f(a)=n$ if $a \in A_{n}$ but $a \notin A_{n-1}$. The

Received by the editors November 25, 1980.

1980 Mathematics Subject Classification. Primary 16A72, 16A05, 16A03, 16A08, 17B35, 17 B40.

${ }^{1}$ Research supported in part by NSF Grant No. MCS 76-07240. 
associated graded ring is $\bar{A}=\sum_{n>0} \oplus A_{n} / A_{n-1}$. We note that $\bar{A}$ is a domain if and only if $f(a b)=f(a)+f(b)$, all $a, b \in A$. When $\bar{A}$ is a domain, so is $A$, and thus $A_{\mathscr{F}}$ is a prime ring.

Proposition 1. Let $A$ be a filtered algebra, as above, such that $\bar{A}$ is a commutative domain. Let $\sigma$ be an $X$-inner automorphism of $A$. Then:

(1) $\sigma$ preserves the filtration of $A$ (that is, $A_{n}^{\sigma}=A_{n}$, all $n$ ),

(2) the induced action $\bar{\sigma}$ of $\sigma$ on $\bar{A}$ is trivial.

Proof. Since $\sigma$ is $X$-inner, we may choose $b, c \in A$ such that

$$
b r c=c r^{\sigma} b^{\sigma}, \text { for all } r \in A \text {. }
$$

Since $\bar{A}$ is a domain, $f(b)+f(r)+f(c)=f(c)+f\left(r^{\sigma}\right)+f\left(b^{\sigma}\right)$ for all $r \in A$; thus $f(b)+f(r)=f\left(r^{\sigma}\right)+f\left(b^{\sigma}\right)$. Letting $r=1=r^{\sigma}, f(r)=f\left(r^{\sigma}\right)=0$, and so $f(b)$ $=f\left(b^{\sigma}\right)$. It follows that $f(r)=f\left(r^{\sigma}\right)$, all $r \in A$. That is, $\sigma$ preserves the filtration.

Now let $k=f(b), l=f(c)$, and let $\bar{b}=b+A_{k-1}, \overline{b^{\sigma}}=b^{\sigma}+A_{k-1}$ be the "leading terms" of $b$ and $b^{\circ}$, respectively. We claim that $\bar{b}=\overline{b^{\circ}}$. For, using $r=1$ in (*), $b c=c b^{\sigma}$. Since $\bar{A}$ is commutative, $c b^{\sigma}-b^{\sigma} c \in A_{k+l-1}$, and thus $\left(b-b^{\sigma}\right) c=$ $c b^{\sigma}-b^{\sigma} c \in A_{k+l-1}$. Since $f(c)=l$, it follows that $f\left(b-b^{\sigma}\right)<k-1$. Thus $b-b^{\sigma} \in A_{k-1}$, so $\bar{b}=\overline{b^{\sigma}}$.

Finally, consider any $x \in A_{n} ; b x c=c x^{\sigma} b^{\sigma}$ from (*), and $c x^{\sigma} b^{\sigma}-b^{\sigma} x^{\sigma} c \in$ $A_{k+l+n-1}$ since $\bar{A}$ is commutative. Thus $\left(b x-b^{\sigma} x^{\sigma}\right) c \in A_{k+l+n-1}$; since $f(c)=l$, we obtain $f\left(b x-b^{\sigma} x^{\sigma}\right) \leqslant k+n-1$, and so $b x-b^{\sigma} x^{\sigma} \in A_{k+n-1}$. Using $b-b^{\sigma}$ $\in A_{k-1}$ from above, $\left(b-b^{\sigma}\right) x^{\sigma} \in A_{k+n-1}$, and so $b\left(x-x^{\sigma}\right)=b x-b^{\sigma} x^{\sigma}+$ $b^{\sigma} x^{\sigma}-b x^{\sigma} \in A_{k+n-1}$. As before, as $f(b)=k$; this forces $x-x^{\sigma} \in A_{n-1}$. Thus $\bar{x}=\overline{x^{\sigma}}$, for any $x \in A_{n}$. We have proved that the induced automorphism $\bar{\sigma}$ (given by $\bar{x}^{\sigma}=\overline{x^{\sigma}}$ ) is trivial on $\bar{A}$.

Our first application of Proposition 1 is to enveloping algebras. Thus, following $[1, \S 4.3]$, let $g$ denote a finite-dimensional Lie algebra over a field $k$, with universal enveloping algebra $U=U(\mathrm{~g})$. Let $K=K(\mathrm{~g})$ denote the division ring of quotients of $U$. For $P$ a completely prime ideal of $U, A=U / P$ also has a division ring of quotients, which we will denote by $Q(A)$.

Let $\varepsilon$ be the adjoint representation of $g$ in $A$; that is if $a \in A$ and $i(x)$ is the image of $x \in g$ in $A$, then $\varepsilon(x) a=[i(x), a]$. For $\lambda \in g^{*}$, let $A_{\lambda}=\{a \in A \mid \varepsilon(x) a=$ $\lambda(x) a$, all $x \in \mathrm{g}\}$. We have $A_{\lambda} A_{\mu} \subset A_{\lambda+\mu}$, and the sum of the $A_{\lambda}$ is direct. The subalgebra $S(A)=\Sigma_{\lambda \in g^{*}} \oplus A_{\lambda}$ is called the semicentre of $A$. More generally, as in 4.9.7 of [1], we may also define $Q(A)_{\lambda}$.

Give $A$ the induced filtration from $U$, and thus $i(g)$ generates $A$. When the associated graded ring $\bar{A}$ of $A=U / P$ is a domain, we are now able to completely determine those automorphisms of $A$ which become inner on $Q(A)$; they are precisely those automorphisms which are given by conjugation by an element of some $Q(A)_{\lambda}$.

THEOREM 1. Let $A=U(\mathrm{~g}) / P$, for $\mathrm{g}$ a finite-dimensional Lie algebra, and assume that $\bar{A}$ is a domain. Let $0 \neq a \in Q(A)$. Then $a^{-1} A a=A \Leftrightarrow$ there exists $\lambda \in \mathrm{g}^{*}$ such that $a \in Q(A)_{\lambda}$. 
Proof. First assume that $a \in Q(A)_{\lambda}$. Then $[i(x), a]=\lambda(x) a$, all $x \in g$, and so $i(x) a=a i(x)+\lambda(x) a=a(i(x)+\lambda(x))$. Thus $i(g) a \subseteq a A$, and it follows that $A a$ $\subseteq a A$. Similarly $a A \subseteq a A$, and so $a A=A a$. It follows that $a^{-1} A a=A$.

Conversely, if $a^{-1} A a=A$, then $\sigma \in \operatorname{Aut}(A)$ given by $r^{\circ}=a^{-1} r a$ is an $X$-inner automorphism of $A$. By Proposition $1, \bar{\sigma}$ is trivial on the associated graded ring $A$. In particular, for any $i(x) \in i(g) \subseteq A_{1}, i(x)^{\sigma}-i(x) \in A_{0}=k \cdot 1$. That is, $i(x)^{\sigma}=$ $i(x)+\lambda(x)$, some $\lambda=\lambda(x) \in k$, for each $x \in g$. Clearly $\lambda \in g^{*}$, and since $i(x)^{\circ}=$ $a^{-1} i(x) a=i(x)+\lambda(x),[i(x), a]=\lambda(x) a$, all $x \in g$. Thus $a \in Q(A)_{\lambda}$.

COROLlary 1. The subgroup of all $X$-inner automorphisms of $A=U(\mathrm{~g}) / P$, for $\bar{A}$ a domain, is isomorphic to the additive subgroup of $\mathrm{g}^{*}$ consisting of those $\lambda$ with $Q(A)_{\lambda} \neq 0$.

Corollary 2. Consider $A=U(\mathrm{~g}) / P$ as above, with $\bar{A}$ a domain, and let $\sigma$ be any automorphism of $A$. Then

(1) $\left(Q(A)_{\lambda}\right)^{\sigma}=Q(A)_{\mu}$, for some $\mu \in g^{*}$.

(2) $\sigma$ stabilizes $S(A)$, the semicentre of $A$.

Proof. Choose any $0 \neq a \in Q(A)_{\lambda}$. By Theorem $1, a^{-1} A a=A$ and thus $\left(a^{\sigma}\right)^{-1} A^{\sigma} a^{\sigma}=A^{\sigma}$, or $\left(a^{\sigma}\right)^{-1} A a^{\sigma}=A$. Thus, again by Theorem 1 , there exists $\mu \in \mathrm{g}^{*}$ such that $a^{\sigma} \in Q(A)_{\mu}$. It follows that $Q(A)_{\lambda}^{\sigma}=Q(A)_{\mu}$, since $\sigma$ preserves the center $C$ of $Q(A)_{\lambda}$ and $Q(A)_{\lambda}=C a$.

Clearly $\sigma$ preserves $S(A)$, since $A_{\lambda}=Q(A)_{\lambda} \cap A$.

We now turn to derivations of $U(\mathrm{~g})$. The next corollary was pointed out to us by Martha Smith, and we wish to thank her for allowing us to include it.

Corollary 3 (M. SMITH). Let $d$ be a derivation of $\mathrm{g}$, a finite-dimensional Lie algebra over a field $k$ of characteristic 0 , and extend $d$ to $U(\mathrm{~g})$. Then for any $\lambda \in \mathrm{g}^{*}$ such that $U_{\lambda} \neq 0$,

(1) $d\left(U_{\lambda}\right) \subseteq U_{\lambda}$,

(2) $\lambda(d(g))=0$.

Proof. We first note that by passing to the algebraic closure of $k$, we may assume that $k$ is algebraically closed. Since $d$ is a locally finite derivation on $U$, and the semicentre $S=S(U)$ is stable under $\operatorname{Aut}(U)$ by Corollary 2, we may apply a result of J. Krempa [2] to conclude that $S$ is also $d$-stable.

Let $0 \neq a \in U_{\lambda}$, some $\lambda \in g^{*}$. Then $d(a) \in S$, and so $d(a)=\Sigma_{\mu} b_{\mu}$, where $b_{\mu} \in U_{\mu}$. Applying $d$ to the equation $[x, a]=\lambda(x) a$, any $x \in g$, we see that $[x, d(a)]=-\lambda(d(x)) a+\lambda(x) d(a)$. Now

$$
\sum_{\mu} \mu(x) b_{\mu}=\sum_{\mu}\left[x, b_{\mu}\right]=[x, d(a)]=-\lambda(d(x)) a+\lambda(x) \sum_{\mu} b_{\mu},
$$

and so

$$
\sum_{\mu}(\mu(x)-\lambda(x)) b_{\mu}=-\lambda(d(x)) a .
$$

For each $u \neq \lambda$, this gives $(\mu(x)-\lambda(x)) b_{\mu}=0$, all $x \in \mathrm{g}$. Since $\lambda\left(x_{0}\right) \neq \mu\left(x_{0}\right)$ for some $x_{0} \in g$, it follows that $b_{\mu}=0$, all $\mu \neq \lambda$. That is, $d(a)=b_{\lambda} \in U_{\lambda}$, proving 
(1). Again using (**), it follows that $0=-\lambda(d(x)) a$. Thus $\lambda(d(x))=0$, all $x \in \mathfrak{g}$, proving (2).

We also give an application to crossed products. For any $k$-algebra $A$, any subgroup $G \subseteq \operatorname{Aut}_{k}(A)$, and any factor set $t: G \times G \rightarrow k$, we may form the crossed product algebra $A *, G$.

COROLlary 4. Let $g$ be a finite-dimensional Lie algebra over $a$ field $k$ of characteristic 0 , and let $A=U(g) / p$ as above, such that $\bar{A}$ is a domain. Let $G$ be any subgroup of $\operatorname{Aut}_{k}(A)$. Then any crossed product $A *_{t} G$ is a prime ring.

Proof. Let $G_{\text {inn }}=\{g \in G \mid g$ is $X$-inner $\}$. By Corollary $1, G_{\text {inn }}$ is isomorphic to a subgroup of the dual space $g^{*}$, which is torsion-free when $k$ has characteristic 0 . Thus the crossed product is prime by [6, Theorem 2.8].

Our second application of Proposition 1 is to certain differential polynomial rings. Let $R$ be a commutative domain with 1 with a nontrivial derivation $d$, and let $A=R[x ; d]$, the differential polynomial ring, in which $x r=r x+d(r)$, for all $r \in R . A$ is a filtered algebra, using $A_{n}=\{$ all polynomials of degree $<n\}$, and the associated graded ring $\bar{A} \cong R[x]$, the ordinary polynomial ring over $R$. We let $F=Q(R)$, the quotient field of $R$, and let $D=Q(A)$, the division ring of quotients of $A$. We are now able to determine all $X$-inner automorphisms of $A$.

TheORem 2. Let $A=R[x ; d]$, where $d$ is a nontrivial derivation of the commutative domain $R$, and let $\sigma \in \operatorname{Aut}(A)$. Then $\sigma$ becomes inner on $D=Q(A) \Leftrightarrow \sigma$ is conjugation by some $q \in F$ such that $q^{-1} d(q) \in R$.

Proof. First assume that $\sigma$ is conjugation by some such $q$. Clearly $R^{\sigma}=R$, and $x^{\sigma}=q^{-1} x q=q^{-1}(q x+d(q))=x+q^{-1} d(q) \in A$. Thus $\sigma$ is an $X$-inner automorphism of $A$.

Conversely, assume that $\sigma$ becomes inner on $D$; say that $\sigma$ is induced by $b(x) a(x)^{-1}$, where $a(x)=a_{m} x^{m}+\cdots+a_{1} x+a_{0}$ and $b(x)=b_{n} x^{n}+\cdots+b_{0}$, $a_{i}, b_{i} \in R$. We will show that $\sigma$ is also induced by $q=b_{n} a_{m}^{-1} \in F$ (the fact that $q^{-1} d(q) \in R$ follows from the fact that $\left.\sigma \in \operatorname{Aut}(A)\right)$.

Now by Proposition 1, we know that $\sigma$ preserves the filtration on $A$ and that $\bar{\sigma}$ is trivial on $\bar{A}$. In particular, $r^{\sigma}=r$, all $r \in R$, and $x^{\sigma}=x+\alpha$, for some $\alpha \in R$.

To simplify the argument, note that $b(x)=b_{1}(x) b_{n}$, so $b(x) a(x)^{-1}=$ $b_{1}(x)\left(b_{n}^{-1} a(x)\right)^{-1}$; that is, we may assume $b(x)$ is monic. Moreover, since for any $f(x) \in A, f(x)^{\sigma}=a(x) b(x)^{-1} f(x) b(x) a(x)^{-1}$, replacing $f$ by $b(x) f$, it follows that $a(x) f(x) b(x)=b(x)^{\sigma} f(x)^{\sigma} a(x)$, all $f(x) \in A$. Again since $\bar{\sigma}$ is trivial on $\bar{A}$, $b(x)^{\sigma}-b(x)$ has degree $<n$; that is, $b(x)^{\sigma}$ is also monic of degree $n$, say $b(x)^{o}=c(x)=x^{n}+\cdots+c_{0}$. Thus

$$
a(x) f(x) b(x)=c(x) f(x)^{\sigma} a(x), \quad \text { all } f \in A .
$$

We first evaluate $(+)$ for $f=r=r^{\sigma}, r \in R$ :

$$
\begin{gathered}
\left(a_{m} x^{m}+a_{m-1} x^{m-1}+\cdots+a_{0}\right) r\left(x^{n}+b_{n-1} x^{n-1}+\cdots+b_{0}\right) \\
=\left(x^{n}+\cdots+c_{0}\right) r\left(a_{m} x^{m}+\cdots+a_{0}\right),
\end{gathered}
$$


so

$$
\begin{aligned}
a_{m} r x^{m+n} & +a_{m} m d(r) x^{m+n-1}+a_{m-1} r x^{m+n-1}+a_{m} r b_{n-1} x^{m+n-1}+\cdots \\
& =r a_{m} x^{m+n}+n d\left(r a_{m}\right) x^{n+m-1}+c_{n-1} r a_{m} x^{m+n-1}+r a_{m-1} x^{n+m-1}+\cdots
\end{aligned}
$$

Equating coefficients of $x^{n+m-1}$, we see

$$
m a_{m} d(r)+a_{m-1} r+a_{m} r b_{n-1}=n d(r) a_{m}+n r d\left(a_{m}\right)+c_{n-1} r a_{m}+r a_{m-1} \text {. }
$$

Cancelling and letting $r=1$,

$$
n d\left(a_{m}\right)=a_{m}\left(b_{n-1}-c_{n-1}\right) .
$$

We also evaluate $(+)$ for $f(x)=x, x^{\sigma}=x+\alpha$ :

$$
\begin{aligned}
\left(a_{m} x^{m}+\cdots+a_{0}\right) x\left(x^{n}+\cdots+b_{0}\right) & \\
= & \left(x^{n}+\cdots+c_{0}\right)(x+\alpha)\left(a_{m} x^{m}+\cdots+a_{0}\right) \\
a_{m} x^{m+n+1}+ & a_{m} b_{n-1} x^{m+n}+a_{m-1} x^{m+n}+\cdots \\
= & a_{m} x^{n+m+1}+(n+1) d\left(a_{m}\right) x^{n+m} \\
& +c_{n-1} a_{m} x^{n+m}+a_{m-1} x^{n+m}+\alpha a_{m} x^{n+m}+\cdots
\end{aligned}
$$

Equating coefficients of $x^{n+m}$, we see

$$
a_{m} b_{n-1}+a_{m-1}=(n+1) d\left(a_{m}\right)+c_{n-1} a_{m}+a_{m-1}+\alpha a_{m}
$$

and thus

$$
a_{m}\left(b_{n-1}-c_{n-1}\right)=(n+1) d\left(a_{m}\right)+\alpha a_{m} .
$$

Substituting $(++)$,

$$
n d\left(a_{m}\right)=(n+1) d\left(a_{m}\right)+\alpha a_{m} .
$$

Thus $\alpha a_{m}=-d\left(a_{m}\right)$, and so $\alpha=-a_{m}^{-1} d\left(a_{m}\right)=a_{m} d\left(a_{m}^{-1}\right)$. Using $q=a_{m}^{-1}$, we have proved that

$$
x^{\sigma}=x+\alpha=x+q^{-1} d(q)=q^{-1} x q .
$$

Since $\sigma$ is determined by its action on $x$, the theorem is proved.

We illustrate Theorems 1 and 2 with several examples.

EXAMPLE 1. Let $\mathfrak{g}$ be the 3-dimensional completely solvable Lie algebra over $\boldsymbol{k}$ with basis $\{x, y, z\}$ such that $[x, y]=y,[x, z]=z$, and $[y, z]=0$. Since $[\mathfrak{g}, \mathfrak{g}]=$ $\langle y, z\rangle$, and any $\lambda \in g^{*}$ such that $K_{\lambda} \neq 0$ must annihilate $[g, g], \lambda(y)=0=\lambda(z)$. Thus $\lambda$ is determined by $\lambda(x)=\alpha \in k$. We claim that $\alpha \in \mathbf{Z}$, an integer. For, if $0 \neq a \in K_{\lambda},[y, a]=0=[z, a]$ and $[x, a]=\alpha a$. Using $U(g) \cong k[y, z][x ; d]$, where $d(y)=y, d(z)=z$, we may assume $a=p q^{-1}$, where $p$ and $q$ are both polynomials in $y$ and $z$. Since for a monomial $m$ in $y$ and $z$ of degree $l, d(m)=\operatorname{lm}, d(a)=\alpha a$ implies $d(p) q-p d(q)=\alpha p q$; it follows that $\alpha \in \mathbf{Z}$. When $\alpha=1$, so $\lambda(x)=1$, both $y, z \in U_{\lambda}$, and induce the $X$-inner automorphism $\sigma \in \operatorname{Aut}(U)$ given by $x^{\sigma}=x+1, y^{\sigma}=y, z^{\sigma}=z$. Thus the group of $X$-inners of $U(\mathrm{~g})$ is $\langle\alpha\rangle$, the infinite cyclic group generated by $\sigma$.

EXAMPLE 2. Consider the Weyl algebra $A_{1}=k[x, y], x y-y x=1$, where $k$ has characteristic 0 . Now $A_{1}=k[y][x ; d]$ where $d(y)=1$, the usual derivative. Then $A_{1}$ has no nontrivial $X$-inner automorphisms. For by Theorem 2, if $\sigma$ is $X$-inner, it 
is induced by some $p(y) / q(y) \in k(y)$ such that $(q(y) / p(y)) d(p(y) / q(y)) \in R=$ $k[y]$. Thus, $\left(q^{\prime} p-q p^{\prime}\right) / p q$ must be a polynomial; this is impossible, as

$$
\operatorname{degree}(p q)>\operatorname{degree}\left(q^{\prime} p-q p^{\prime}\right) \text {. }
$$

However, enlarging $A_{1}$ slightly to $B=k[y]_{(y)}[x ; d]$, the localization of $A_{1}$ at the powers of $y$, the group of $X$-inners of $B$ is $\langle\sigma\rangle$, the infinite cyclic group generated by $\sigma$ which is given by conjugation by $y$. For, by Theorem 2, any $X$-inner automorphism $\tau$ is induced by $p y^{k} / q \in k(y)$, where $p, q \in k[y]$ are relatively prime, $y \nmid p, y \nmid q$, and $k \in \mathbf{Z}$. Since conjugation by $y^{k}$ is easily seen to be $X$-inner (it preserves $\left.k[y]_{(y)}\right)$ and the $X$-inner automorphisms form a subgroup, $\tau_{1}=\tau \sigma^{-k}$ is $X$-inner and is induced by $p / q$. As before, $(q / p) d(p / q)=\left(q^{\prime} p-q p^{\prime}\right) / p q \in$ $k[y]_{(y)}$, and this is impossible as $y \nmid p q$, unless $q^{\prime}=p^{\prime}=0$, so $p / q=\alpha \in k$. Thus, $\tau$ is induced by $y^{k}$.

Finally, if we consider $C=k(y)[x ; d]$, then conjugation by any nonzero element of $F=k(y)$ is an $X$-inner automorphism of $C$, and $p, q \in F$ induce the same automorphism $\Leftrightarrow p=\alpha q, \alpha \in k$. Thus the group of $X$-inners is isomorphic to $k(y)^{\circ} / k^{\circ}$, where $K^{\circ}$ denotes the multiplicative group.

\section{REFERENCES}

1. J. Dixmier, Enveloping algebras, North-Holland Math. Library, Vol. 14, Amsterdam, 1977.

2. J. Krempa, On invariant subspaces of locally finite derivations, Bull. London Math. Soc. 12 (1980), 374-376.

3. W. S. Martindale III, Prime rings satisfying a generalized polynomial identity, J. Algebra 12 (1969), 576-584.

4. W. S. Martindale and S. Montgomery, The normal closure of coproducts of domains, J. Algebra (to appear).

5. S. Montgomery, Outer automorphisms of semiprime rings, J. London Math. Soc. 18 (1978), 209-221.

6. S. Montgomery and D. S. Passman, Crossed products over prime rings, Israel J. Math. 31 (1978), $224-256$.

7. X-inner automorphisms of group rings, Houston J. Math. (to appear).

Department of Mathematics, Universtty of Southern California, los Angeles, California 90007 